\title{
Perfect State Transfer on Cayley Graphs over Dihedral Groups: The Non-Normal Case
}

\author{
Xiwang Cao \\ School of Mathematical Sciences \\ Nanjing University of Aeronautics and Astronautics \\ Nanjing 210016, China \\ State Key Laboratory of Information Security \\ Institute of Information Engineering \\ Chinese Academy of Sciences, Beijing 100093, China \\ xwcao@nuaa.edu.cn \\ Bocong Chen \\ School of Mathematics \\ South China University of Technology \\ Guangzhou 510641, China \\ bocong_chen@yahoo.com \\ San Ling \\ Division of Mathematical Sciences \\ School of Physical and Mathematical Sciences \\ Nanyang Technological University \\ Singapore 639798 \\ lingsan@ntu.edu.sg \\ Submitted: Dec 1, 2019; Accepted: May 8, 2020; Published: May 29, 2020 \\ (C) The authors. Released under the CC BY-ND license (International 4.0).

\begin{abstract}
Recently, perfect state transfer (PST for short) on graphs has attracted great attention due to their applications in quantum information processing and quantum computations. Many constructions and results have been established through various graphs. However, most of the graphs previously investigated are abelian Cayley graphs. Necessary and sufficient conditions for Cayley graphs over dihedral groups having perfect state transfer were studied recently. The key idea in that paper is the assumption of the normality of the connection set. In those cases, viewed as an element in a group algebra, the connection set is in the center of the group algebra, which makes the situations just like in the abelian case. In this paper, we study the non-normal case. In this case, the discussion becomes more complicated. Using the representations of the dihedral group $D_{n}$, we show that Cay $\left(D_{n}, S\right)$ cannot have PST if $n$ is odd. For even integers $n$, it is proved that if Cay $\left(D_{n}, S\right)$ has PST, then $S$ is normal.
\end{abstract}

Mathematics Subject Classifications: 05C25 81P45 81Q35 


\section{Introduction}

Let $\Gamma$ be an undirected simple graph whose vertex set is denoted by $V(\Gamma)$. Let $A$ be an adjacency matrix of $\Gamma$. For an real number $t$, the transfer matrix of $\Gamma$ is defined as the following $n \times n$ matrix:

$$
H(t)=H_{\Gamma}(t)=\exp (-\imath t A)=\sum_{s=0}^{+\infty} \frac{(-\imath t A)^{s}}{s !}=\left(H_{u, v}(t)\right)_{u, v \in V(\Gamma)},
$$

where $\imath=\sqrt{-1}$ and $n=|V(\Gamma)|$ is the number of vertices in $\Gamma$.

Definition 1. Let $\Gamma$ be a graph. For two distinct vertices $u, v \in V(\Gamma)$, we say that $\Gamma$ has a perfect state transfer (PST) from $u$ to $v$ at the time $t(>0)$ if the $(u, v)$-entry of $H(t)$, denoted by $H(t)_{u, v}$, has absolute value 1 . We say that $\Gamma$ is periodic at $u$ with period $t$ if $H(t)_{u, u}$ has absolute value 1 . If $\Gamma$ is periodic with period $t$ at every point, then $\Gamma$ is said to be periodic.

The phenomenon of perfect state transfer (PST) in quantum communication networks was originally introduced by Bose in [14]. This work has attracted much research interest since many applications have been found in quantum information processing and cryptography (see $[1,2,3,5,11,15,17,18,34,38,37]$ and the references therein).

For perfect state transfer and related questions such as the close relationship with algebraic combinatorial objects, we refer the reader to Godsil [23, 24, 25] and Coutinho [19] and the references therein.

Since the adjacency matrix $A$ is symmetric, its eigenvalues are all real numbers. A graph $\Gamma$ is called integral if all eigenvalues of the adjacency matrix $A$ are integers [26]. For abelian Cayley graphs, a complete characterization of integral graphs over abelian groups was obtained by Bridges and Mena [12] in 1982.

Despite many results about PST on graphs, there are only a few known constructions of PST on non-abelian Cayley graphs in the literature [13]. In [13], the authors studied the existence of PST on Cayley graphs over dihedral groups. Some necessary and sufficient conditions for a normal Cayley graph Cay $\left(D_{n}, S\right)$ (which means that $S$ is conjugationclosed, namely, $g^{-1} S g=S$ for each $g \in G$ ) having PST were presented. As an application, it was proved that $\operatorname{Cay}\left(D_{n}, S\right)$ is periodic if and only if it is integral. Meanwhile, it was showed that Cay $\left(D_{n}, S\right)$ has PST for some connection set $S$ and some even integers $n$.

In the present paper, we consider the general case. Actually, it is not an easy task to get an explicit expression for the spectra and eigenspaces of Cayley graphs over non-abelian groups when the connection set is non-normal. If the underlying group is a dihedral group, then we show that one can use the Fourier transform to obtain the spectra and eigenvectors of the Cayley graph. Based on this observation, it is proved that Cay $\left(D_{n}, S\right)$ cannot have PST if $n$ is odd. For even integers $n$, it is shown that Cay $\left(D_{n}, S\right)$ has PST if and only if $S$ is normal.

The rest of the paper is organised as follows: In Sect. 2, we recall some basic facts about finite group representations and the Fourier transform on finite groups. In Sect. 3, 
we provide the spectra and the corresponding eigenspace of Cayley graphs over dihedral groups. In Sect. 4, we present our main results. In Sect. 6, we make some concluding remarks.

\section{The representation of groups and The Fourier Transform}

Let $G$ be a finite group. A representation of $G$ is a homomorphism $\rho: G \rightarrow G L(U)$, where $U$ is a (finite-dimensional) non-zero vector space over the field of complex numbers $\mathbb{C}$. The dimension of $U$ is called the degree of $\rho$. Two representations $\rho: G \rightarrow G L(U)$ and $\varrho: G \rightarrow G L(W)$ are equivalent, denoted by $\rho \sim \varrho$, if there exists an isomorphism $T: U \rightarrow W$ such that $\rho_{g}=T \varrho_{g} T^{-1}$ for all $g \in G$. For a representation $\rho: G \rightarrow G L(U)$ of $G$, the character of $\rho$ is defined by:

$$
\chi_{\rho}: G \rightarrow \mathbb{C}, \chi_{\rho}(g)=\operatorname{tr}(\rho(g)) \text { for all } g \in G,
$$

where $\operatorname{tr}(\rho(g))$ is the trace of the matrix $\rho(g)$ with respect to a basis of $U$. A subspace $W$ of $U$ is said to be $G$-invariant if $\rho(g) \omega \in W$ for every $g \in G$ and $\omega \in W$. Obviously, $\{0\}$ and $U$ are $G$-invariant subspaces, which are called trivial subspaces. If $U$ has no non-trivial $G$-invariant subspaces, then $\rho$ is called an irreducible representation of $G$ and $\chi_{\rho}$ is called an irreducible character of $G$.

Let $S$ be a subset of $G$ with $|S|=d \geqslant 1$. The Cayley graph $\Gamma=\operatorname{Cay}(G, S)$ is defined by

$$
\begin{aligned}
& V(\Gamma)=G, \text { the set of vertices, } \\
& E(\Gamma)=\left\{(u, v): u, v \in G, u v^{-1} \in S\right\}, \text { the set of edges. }
\end{aligned}
$$

We assume that the identity element of $G$ is not belonged to $S$ (denoted by $1_{G} \notin S$ ), $S=S^{-1}=\left\{s^{-1}: s \in S\right\}$ (which means that $\Gamma$ is a simple graph) and $G=\langle S\rangle(G$ is generated by $S$ which means that $\Gamma$ is connected). The adjacency matrix of $\Gamma$ is defined by $A=A(\Gamma)=\left(a_{g, h}\right)_{g, h \in G}$ where

$$
a_{g, h}=\left\{\begin{array}{lc}
1, & \text { if } g h^{-1} \in E(\Gamma) \\
0, & \text { otherwise }
\end{array}\right.
$$

Let $L(G)=\{f: G \rightarrow \mathbb{C}\}$ be the set of complex-valued functions from $G$ to $\mathbb{C}$. For $f_{1}, f_{2} \in L(G)$, we define

$$
\left(f_{1} * f_{2}\right)(g)=\sum_{h \in G} f_{1}\left(g h^{-1}\right) f_{2}(h) .
$$

Then $L(G)$ is a $\mathbb{C}$-algebra. Moreover, we have a scalar product $\langle\cdot, \cdot\rangle_{L(G)}$ on $L(G)$ by setting

$$
\left\langle f_{1}, f_{2}\right\rangle_{L(G)}=\sum_{g \in G} f_{1}(g) \overline{f_{2}(g)}
$$


where $^{-}$is the conjugate. For every $g \in G$, we define a mapping $\delta_{g} \in L(G)$ by

$$
\delta_{g}(x)= \begin{cases}1, & x=g \\ 0, & x \neq g\end{cases}
$$

Then it is easy to see that $\left\{\delta_{g}: g \in G\right\}$ forms a basis of $L(G)$ and thus $\operatorname{dim}(L(G))=|G|$. Define $f_{S} \in \operatorname{Hom}(L(G), L(G))$ by

$$
f_{S}: L(G) \rightarrow L(G), \sum_{g \in G} c_{g} \delta_{g} \mapsto \sum_{s \in S} \sum_{g \in G} c_{g} \delta_{s g}
$$

The matrix of $f_{S}$ under the basis $\left\{\delta_{g}: g \in G\right\}$ is identical to the adjacent matrix of the Cayley graph $\operatorname{Cay}(G, S)$.

From now on, we focus on the unitary representations of $G$. Let $\hat{G}$ be a complete set of pairwise inequivalent irreducible unitary representations of $G$. Let $\mathcal{A}(G)$ be defined as

$$
\mathcal{A}(G)=\bigoplus_{\rho \in \hat{G}} \operatorname{Hom}\left(W_{\rho}, W_{\rho}\right)
$$

where $W_{\rho}$ is the representation space corresponding to the unitary irreducible representation $\rho$. Suppose that $\left\{v_{1}^{\rho}, \cdots, v_{d_{\rho}}^{\rho}\right\}$ is an orthonormal basis of $W_{\rho}$ for any $\rho \in \hat{G}$. For $\rho, \sigma \in \hat{G}$ and $1 \leqslant i, j \leqslant d_{\rho}$, define

$$
T_{i, j}^{\rho} \omega=\delta_{\rho, \sigma}\left\langle\omega, v_{j}^{\rho}\right\rangle_{W_{\rho}} v_{i}^{\rho}, \omega \in W_{\sigma},
$$

where $\delta_{\rho, \sigma}=1$ if $\rho \sim \sigma$ and 0 otherwise. Then $T_{i, j}^{\rho} \in \mathcal{A}(G)$ and $\left\{T_{i, j}^{\rho}: \rho \in \hat{G}, 1 \leqslant i, j \leqslant d_{\rho}\right\}$ form a basis of $\mathcal{A}(G)$. In correspondence to the basis $\left\{v_{1}^{\rho}, \cdots, v_{d_{\rho}}^{\rho}\right\}$ of $W_{\rho}$, we define

$$
\varphi_{i, j}^{\rho}: G \rightarrow \mathbb{C}, g \mapsto\left\langle\rho(g) v_{j}^{\rho}, v_{i}^{\rho}\right\rangle_{W_{\rho}} .
$$

Then $\varphi_{i, j}^{\rho} \in L(G)$ and it is known that $\left\{\varphi_{i, j}^{\rho}: \rho \in \hat{G}, 1 \leqslant i, j \leqslant d_{\rho}\right\}$ forms an orthonormal basis of $L(G)$. For every $f \in L(G)$, the Fourier transform of $f$ is defined by

$$
\mathcal{F}(f)=\bigoplus_{\rho \in \hat{G}} \rho(f)
$$

where $\rho(f)$ is defined as

$$
\rho(f): W_{\rho} \rightarrow W_{\rho}, \omega \mapsto \sum_{g \in G} f(g) \rho(g)(\omega)
$$

In other words, $\rho(f)=\sum_{g \in G} f(g) \rho(g)$ and thus $\rho(f) \in \operatorname{Hom}\left(W_{\rho}, W_{\rho}\right)$. It is known that the Fourier transform $\mathcal{F}$ is an algebra isomorphism from $L(G)$ to $\mathcal{A}(G)$ and

$$
\overline{\mathcal{F}} \overline{\varphi_{i, j}^{\rho}}=\frac{|G|}{d_{\rho}} T_{i, j}^{\rho}
$$


See [35] for details. Thus we have the following commutative diagram:

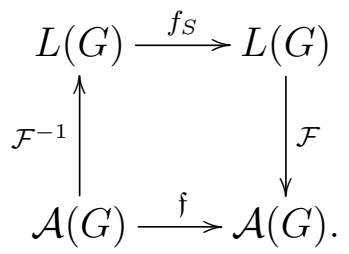

Moreover,

$$
\mathfrak{f}\left(T_{i, j}^{\rho}\right)=\frac{d \rho}{|G|} \mathcal{F} f_{S} \overline{\varphi_{i, j}^{\rho}}
$$

Now,

$$
\begin{aligned}
f_{S} \overline{\varphi_{i, j}^{\rho}}(g) & =\sum_{s \in S} \overline{\varphi_{i, j}^{\rho}}\left(s^{-1} g\right) \\
& =\sum_{s \in S} \overline{\left\langle\rho\left(s^{-1} g\right) v_{j}^{\rho}, v_{i}^{\rho}\right\rangle} \\
& =\sum_{s \in S} \overline{\left\langle\rho(g) v_{j}^{\rho}, \rho(s) v_{i}^{\rho}\right\rangle} \\
& =\sum_{s \in S}\left\langle\rho(g) v_{j}^{\rho}, \sum_{k=1}^{d_{\rho}}\left\langle\rho(s) v_{i}^{\rho}, v_{k}^{\rho}\right\rangle v_{k}^{\rho}\right\rangle \\
& =\sum_{s \in S} \sum_{k=1}^{d_{\rho}}\left\langle\rho(s) v_{i}^{\rho}, v_{k}^{\rho}\right\rangle \overline{\left\langle\rho(g) v_{j}^{\rho}, v_{k}^{\rho}\right\rangle} \\
& =\sum_{k=1}^{d_{\rho}}\left\langle\sum_{s \in S} \rho(s) v_{i}^{\rho}, v_{k}^{\rho}\right\rangle \overline{\left\langle\rho(g) v_{j}^{\rho}, v_{k}^{\rho}\right\rangle} \\
& =\sum_{k=1}^{d_{\rho}}\left\langle\sum_{s \in S} \rho(s) v_{i}^{\rho}, v_{k}^{\rho}\right\rangle \overline{\varphi_{k, j}^{\rho}(g)} .
\end{aligned}
$$

Therefore,

$$
\mathfrak{f}\left(T_{i, j}^{\rho}\right)=\frac{d_{\rho}}{|G|} \mathcal{F} f_{S} \overline{\varphi_{i, j}^{\rho}}=\frac{d_{\rho}}{|G|} \sum_{k=1}^{d_{\rho}}\left\langle\sum_{s \in S} \rho(s) v_{i}^{\rho}, v_{k}^{\rho}\right\rangle \overline{\mathcal{F}} \overline{\varphi_{k, j}^{\rho}}
$$

That is

$$
\mathfrak{f}\left(T_{i, j}^{\rho}\right)=\sum_{k=1}^{d_{\rho}}\left\langle\sum_{s \in S} \rho(s) v_{i}^{\rho}, v_{k}^{\rho}\right\rangle T_{k, j}^{\rho}, \rho \in \hat{G}, 1 \leqslant i, j \leqslant d_{\rho} .
$$

If $S$ is conjugation-closed, namely $g S g^{-1}=S$ for all $g \in S$, then by the Schur orthogonality relations ([36, Theorem 4.2.8]) we have the following result. 
Lemma 2. [36, pp. 69-70] Let $G=\left\{g_{1}, \cdots, g_{n}\right\}$ be a finite group of order $n$ and let $\rho^{(1)}, \cdots, \rho^{(s)}$ be a complete set of unitary representatives of the equivalent classes of irreducible representations of $G$. Let $\chi_{i}$ be the character of $\rho^{(i)}$ and let $d_{i}$ be the degree of $\chi_{i}$. Let $S$ be a subset of $G$ with $1_{G} \notin S, S=S^{-1}$ and $g S g^{-1}=S$ for all $g \in G$. Then the eigenvalues of the adjacency matrix $A$ of the Cayley graph $\operatorname{Cay}(G, S)$ with respect to $S$ are given by

$$
\lambda_{k}=\frac{1}{d_{k}} \sum_{g \in S} \chi_{k}(g), 1 \leqslant k \leqslant s,
$$

where each $\lambda_{k}$ has multiplicity $d_{k}^{2}$. Moreover, the vectors

$$
v_{i j}^{(k)}=\frac{\sqrt{d_{k}}}{|G|}\left(\rho_{i j}^{(k)}\left(g_{1}\right), \cdots, \rho_{i j}^{(k)}\left(g_{n}\right)\right)^{T}, 1 \leqslant i, j \leqslant d_{k}
$$

form an orthonormal basis for the eigenspace $V_{\lambda_{k}}$.

Note that a proof of Lemma 2 can also be found in [31, Theorem 9]. Babai [7] studied the spectra of Cayley color graphs, which generalizes the notion of Cayley graphs; however, [7] does not consider how to find the corresponding eigenvectors of the eigenvalues. If $S$ is not conjugation-closed, to the best of our knowledge, no method in literature tells how one can find the eigenvalues and the corresponding eigenvectors of the Cayley graphs. In the next section, we will use (1) to find the eigenvalues and eigenvectors of $f_{S}$.

\section{Spectra and the corresponding eigenspaces of Cayley graphs over dihedral groups}

For the dihedral group $D_{n}=\left\langle a, b \mid a^{n}=b^{2}=1, b a b=a^{-1}\right\rangle$, its representations and characters are well known as given below.

Lemma 3. [33, pp. 36-37] (1) The irreducible representations of the dihedral group $D_{n}(n \geqslant 2)$ are listed in Table 1 for even $n$ and in Table 2 for odd $n$.

\begin{tabular}{|c|c|c|}
\multicolumn{2}{c}{ Table 1. Representations of $D_{n}$ (n even) } \\
\hline & $a^{k}(0 \leqslant k \leqslant n-1)$ & $b a^{k}(0 \leqslant k \leqslant n-1)$ \\
\hline$\psi_{1}$ & 1 & 1 \\
$\psi_{2}$ & 1 & -1 \\
$\psi_{3}$ & $(-1)^{k}$ & $(-1)^{k}$ \\
$\psi_{4}$ & $(-1)^{k}$ & $(-1)^{k+1}$ \\
$\rho_{h}$ & $\left(\begin{array}{cc}\omega^{h k} & 0 \\
0 & \omega^{-h k}\end{array}\right)$ & $\left(\begin{array}{cc}0 & \omega^{-h k} \\
\omega^{h k} & 0\end{array}\right)$ \\
\hline
\end{tabular}


Table 2. Representations of $D_{n}(n$ odd $)$

\begin{tabular}{|c|c|c|}
\hline & $a^{k}(0 \leqslant k \leqslant n-1)$ & $b a^{k}(0 \leqslant k \leqslant n-1)$ \\
\hline$\psi_{1}$ & 1 & 1 \\
$\psi_{2}$ & 1 & -1 \\
$\rho_{h}$ & $\left(\begin{array}{cc}\omega^{h k} & 0 \\
0 & \omega^{-h k}\end{array}\right)$ & $\left(\begin{array}{cc}0 & \omega^{-h k} \\
\omega^{h k} & 0\end{array}\right)$ \\
\hline
\end{tabular}

(2) The character table of $D_{n}$ is listed in Table 3 for even $n$ and in Table 4 for odd $n$.

Table 3. Character table of $D_{n}$ ( $n$ even)

\begin{tabular}{|c|c|c|}
\hline & $a^{k}(0 \leqslant k \leqslant n-1)$ & $b a^{k}(0 \leqslant k \leqslant n-1)$ \\
\hline$\chi_{1}$ & 1 & 1 \\
$\chi_{2}$ & 1 & -1 \\
$\chi_{3}$ & $(-1)^{k}$ & $(-1)^{k}$ \\
$\chi_{4}$ & $(-1)^{k}$ & $(-1)^{k+1}$ \\
$\chi_{h}$ & $2 \cos \left(\frac{2 h k \pi}{n}\right)$ & 0 \\
$1 \leqslant h \leqslant\left\lfloor\frac{n-1}{2}\right\rfloor$ & & \\
\hline
\end{tabular}

Table 4. Character table of $D_{n}(n$ odd $)$

\begin{tabular}{|c|c|c|}
\hline & $a^{k}(0 \leqslant k \leqslant n-1)$ & $b a^{k}(0 \leqslant k \leqslant n-1)$ \\
\hline$\chi_{1}$ & 1 & 1 \\
$\chi_{2}$ & 1 & -1 \\
$\chi_{h}$ & $2 \cos \left(\frac{2 h k \pi}{n}\right)$ & 0 \\
$1 \leqslant h \leqslant\left\lfloor\frac{n-1}{2}\right\rfloor$ & & \\
\hline
\end{tabular}

where $\omega=\exp \left(\frac{2 \pi \imath}{n}\right)$ is a primitive $n$-th root of unity.

Let Cay $\left(D_{n}, S\right)$ be a Cayley graph with $1_{G} \notin S, S=S^{-1}$ and $G=D_{n}=\langle S\rangle$. Let $S_{1}=S \cap\langle a\rangle$ and $b S_{2}=S \cap b\langle a\rangle$. We assume that $s_{1}=\left|S_{1}\right|, s_{2}=\left|S_{2}\right|$ and $d=s_{1}+s_{2}=|S|$.

\section{1 $n$ is odd}

We first consider the one-dimensional representations, and we obtain the following simple result.

Lemma 4. For the one-dimensional representations of $D_{n}$, one has

(1) The eigenvalue corresponding to the trivial representation is $\lambda_{1}=d=|S|$, and the associated eigenvector is

$$
p_{1}=\frac{1}{\sqrt{2 n}} \varphi_{11}^{\psi_{1}}=\frac{1}{\sqrt{2 n}}(1,1, \cdots, 1)^{t} .
$$

(2) The eigenvalue corresponding to the one-dimensional representation $\psi_{2}$ is $\lambda_{2}=$ $s_{1}-s_{2}$, and the associated eigenvector is

$$
p_{2}=\frac{1}{\sqrt{2 n}} \varphi_{11}^{\psi_{2}}=\frac{1}{\sqrt{2 n}}(\underbrace{1,1, \cdots,}_{n \text { times }}, \underbrace{-1,-1, \cdots,-1}_{n \text { times }})^{t} .
$$


Proof. Now, suppose that $\rho=\psi_{1}$ is the trivial representation. By (1), we have

$$
\mathfrak{f}\left(T_{i, j}^{\psi_{1}}\right)=\sum_{k=1}^{d_{\psi_{1}}}\left\langle\sum_{s \in S} \rho(s) v_{i}^{\psi_{1}}, v_{k}^{\psi_{1}}\right\rangle T_{k, j}^{\psi_{1}}=d T_{i, j}^{\psi_{1}}, 1 \leqslant i, j \leqslant d_{\psi_{1}}=1 .
$$

This implies that

$$
f_{S} \mathcal{F}^{-1} T_{i, j}^{\psi_{1}}=\sum_{k=1}^{d_{\psi_{1}}}\left\langle\sum_{s \in S} \rho(s) v_{i}^{\psi_{1}}, v_{k}^{\psi_{1}}\right\rangle \mathcal{F}^{-1} T_{k, j}^{\psi_{1}}=d \mathcal{F}^{-1} T_{i, j}^{\psi_{1}}, \quad 1 \leqslant i, j \leqslant d_{\psi_{1}}=1 .
$$

We have shown that $d$ is the eigenvalue corresponding to the trivial representation and the associated eigenvector is $p_{1}=\frac{1}{\sqrt{2 n}} \varphi_{11}^{\psi_{1}}=\frac{1}{\sqrt{2 n}}(1,1, \cdots, 1)^{t}$.

Next we assume that $\rho=\psi_{2}$. Then

$$
\mathfrak{f}\left(T_{i, j}^{\psi_{2}}\right)=\sum_{k=1}^{d_{\psi_{2}}}\left\langle\sum_{s \in S} \rho(s) v_{i}^{\psi_{2}}, v_{k}^{\psi_{2}}\right\rangle T_{k, j}^{\psi_{2}}=\left(s_{1}-s_{2}\right) T_{i, j}^{\psi_{2}}, 1 \leqslant i, j \leqslant d_{\psi_{2}}=1 .
$$

It follows that the eigenvalue corresponding to $\psi_{2}$ is $s_{1}-s_{2}$ and the associated eigenvector is

$$
\frac{1}{\sqrt{2 n}}(\underbrace{1,1, \cdots, 1}_{n \text { times }}, \underbrace{-1,-1, \cdots,-1}_{n \text { times }})^{t} .
$$

Suppose now that $\rho_{h}$ is a two-dimensional representation for $1 \leqslant h \leqslant\left\lfloor\frac{n-1}{2}\right\rfloor$. We then have

$$
\left\{\begin{array}{l}
\rho_{h}\left(a^{k}\right) v_{1}^{\rho_{h}}=\omega^{h k} v_{1}^{\rho_{h}}, \\
\rho_{h}\left(a^{k}\right) v_{2}^{\rho_{h}}=\omega^{-h k} v_{2}^{\rho_{h}}, \\
\rho_{h}(b) v_{1}^{\rho_{h}}=v_{2}^{\rho_{h}} \\
\rho_{h}(b) v_{2}^{\rho_{h}}=v_{1}^{\rho_{h}} .
\end{array}\right.
$$

Let

$$
\eta_{h}\left(S_{1}\right)=\sum_{a^{k} \in S_{1}} \omega^{h k}, \eta_{h}\left(S_{2}\right)=\sum_{a^{k} \in S_{2}} \omega^{h k} .
$$

Assume that, corresponding to the two-dimensional representation $\rho_{h}, \mu_{h}$ is an eigenvalue of $f_{S}$ associated with an eigenvector $v_{\rho_{h}}$.

Lemma 5. Let the notation be defined as above. The eigenvalues corresponding to the representation $\rho_{h}$ are

$$
\mu_{h}=\eta_{h}\left(S_{1}\right) \pm \sqrt{\eta_{h}\left(S_{2}\right) \eta_{h}\left(S_{2}^{-1}\right)},\left(1 \leqslant h \leqslant\left\lfloor\frac{n-1}{2}\right\rfloor\right),
$$

each with multiplicity 2. 
Proof. By (1) and (2), we have

$$
\left\{\begin{array}{l}
\mathfrak{f}\left(T_{11}^{\rho_{h}}\right)=\eta_{h}\left(S_{1}\right) T_{11}^{\rho_{h}}+\eta_{h}\left(S_{2}\right) T_{21}^{\rho_{h}} \\
\mathfrak{f}\left(T_{12}^{\rho_{h}}\right)=\eta_{h}\left(S_{1}\right) T_{12}^{\rho_{h}}+\eta_{h}\left(S_{2}\right) T_{22}^{\rho_{h}} \\
\mathfrak{f}\left(T_{21}^{\rho_{h}}\right)=\eta_{h}\left(S_{2}^{-1}\right) T_{11}^{\rho_{h}}+\eta_{h}\left(S_{1}\right) T_{21}^{\rho_{h}} \\
\mathfrak{f}\left(T_{22}^{\rho_{h}}\right)=\eta_{h}\left(S_{2}^{-1}\right) T_{12}^{\rho_{h}}+\eta_{h}\left(S_{1}\right) T_{22}^{\rho_{h}}
\end{array}\right.
$$

In matrix form, we have

$$
\mathfrak{f}\left(T_{11}^{\rho_{h}}, T_{21}^{\rho_{h}}, T_{12}^{\rho_{h}}, T_{22}^{\rho_{h}}\right)=\left(T_{11}^{\rho_{h}}, T_{21}^{\rho_{h}}, T_{12}^{\rho_{h}}, T_{22}^{\rho_{h}}\right)\left(\begin{array}{cccc}
\eta_{h}\left(S_{1}\right) & \eta_{h}\left(S_{2}^{-1}\right) & 0 & 0 \\
\eta_{h}\left(S_{2}\right) & \eta_{h}\left(S_{1}\right) & 0 & 0 \\
0 & 0 & \eta_{h}\left(S_{1}\right) & \eta_{h}\left(S_{2}^{-1}\right) \\
0 & 0 & \eta_{h}\left(S_{2}\right) & \eta_{h}\left(S_{1}\right)
\end{array}\right) .
$$

Thus we get the desired result.

By (1), we can assume that $v_{\rho_{h}}=a_{11} T_{11}^{\rho_{h}}+a_{12} T_{12}^{\rho_{h}}+a_{21} T_{21}^{\rho_{h}}+a_{22} T_{22}^{\rho_{h}}$ with $a_{i j} \in \mathbb{C}$. It follows that

$$
\mathfrak{f}\left(a_{11} T_{11}^{\rho_{h}}+a_{12} T_{12}^{\rho_{h}}+a_{21} T_{21}^{\rho_{h}}+a_{22} T_{22}^{\rho_{h}}\right)=\mu_{h}\left(a_{11} T_{11}^{\rho_{h}}+a_{12} T_{12}^{\rho_{h}}+a_{21} T_{21}^{\rho_{h}}+a_{22} T_{22}^{\rho_{h}}\right) .
$$

This leads to

$$
f_{S}\left(a_{11} \varphi_{11}^{\rho_{h}}+a_{12} \varphi_{12}^{\rho_{h}}+a_{21} \varphi_{21}^{\rho_{h}}+a_{22} \varphi_{22}^{\rho_{h}}\right)=\mu_{h}\left(a_{11} \varphi_{11}^{\rho_{h}}+a_{12} \varphi_{12}^{\rho_{h}}+a_{21} \varphi_{21}^{\rho_{h}}+a_{22} \varphi_{22}^{\rho_{h}}\right) .
$$

By solving this equation, we get the following result.

Lemma 6. (1) If $\eta_{h}\left(S_{2}\right)=0$, then the corresponding eigenvalue is $\mu_{h}=\eta_{h}\left(S_{1}\right)$ with multiplicity 4 and the associated eigenvectors are $\varphi_{11}^{\rho_{h}}, \varphi_{21}^{\rho_{h}}, \varphi_{12}^{\rho_{h}}, \varphi_{22}^{\rho_{h}}$.

(2) If $\eta_{h}\left(S_{2}\right) \neq 0$, let $\ell_{h}=\sqrt{\frac{\eta_{h}\left(S_{2}^{-1}\right)}{\eta_{h}\left(S_{2}\right)}}$ and $\iota_{h}=\ell_{h} \bar{\ell}_{h} n+n$. Then the eigenvectors associated with $\mu_{h}^{(1)}=\eta_{h}\left(S_{1}\right)+\sqrt{\eta_{h}\left(S_{2}\right) \eta_{h}\left(S_{2}^{-1}\right)}$ are

$$
p_{h}^{(1)}=\frac{1}{\sqrt{\iota_{h}}}\left(\bar{\ell}_{h} \varphi_{11}^{\rho_{h}}+\varphi_{21}^{\rho_{h}}\right), p_{h}^{(2)}=\frac{1}{\sqrt{\iota_{h}}}\left(\bar{\ell}_{h} \varphi_{12}^{\rho_{h}}+\varphi_{22}^{\rho_{h}}\right) .
$$

The eigenvectors associated with $\mu_{h}^{(2)}=\eta_{h}\left(S_{1}\right)-\sqrt{\eta_{h}\left(S_{2}\right) \eta_{h}\left(S_{2}^{-1}\right)}$ are

$$
p_{h}^{(3)}=\frac{1}{\sqrt{\iota_{h}}}\left(-\varphi_{11}^{\rho_{h}}+\ell_{h} \varphi_{21}^{\rho_{h}}\right), p_{h}^{(4)}=\frac{1}{\sqrt{\iota_{h}}}\left(-\varphi_{12}^{\rho_{h}}+\ell_{h} \varphi_{22}^{\rho_{h}}\right) .
$$

Proof. These facts are deduced from (5) and (6). 


\section{$3.2 n=2 m$ is even}

In this case, Lemmas 4-6 also can be applied in this case, but there are two additional onedimensional representations, namely $\psi_{3}, \psi_{4}$, see Lemma 3. For these two representations, based on the above discussion, we have the following result.

Lemma 7. Let

$S_{10}=\left\{a^{k}: 1 \leqslant k \leqslant n-1, a^{k} \in S_{1}, k\right.$ even $\}, S_{11}=\left\{a^{k}: 1 \leqslant k \leqslant n-1, a^{k} \in S_{1}, k\right.$ odd $\}$ and

$S_{20}=\left\{a^{k}: 1 \leqslant k \leqslant n-1, a^{k} \in S_{2}, k\right.$ even $\}, S_{21}=\left\{a^{k}: 1 \leqslant k \leqslant n-1, a^{k} \in S_{2}, k\right.$ odd $\}$.

Then $S_{1}=S_{10} \cup S_{11}, S_{2}=S_{20} \cup S_{21}$. Let $d_{i 0}=\left|S_{i 0}\right|, d_{i 1}=\left|S_{i 1}\right|, i=1,2$. The eigenvalue corresponding to the representation $\psi_{3}$ is $\lambda_{3}=d_{11}-d_{10}+d_{21}-d_{20}$, and the associated eigenvector is

$$
p_{3}=\frac{1}{\sqrt{2 n}}\left((-1)^{i}: 0 \leqslant i \leqslant 2 m-1,(-1)^{i}: 0 \leqslant i \leqslant 2 m-1\right)^{t} .
$$

The eigenvalue corresponding to the representation $\psi_{4}$ is $\lambda_{4}=d_{11}-d_{10}+d_{20}-d_{21}$, and the associated eigenvector is

$$
p_{4}=\frac{1}{\sqrt{2 n}}\left((-1)^{i}: 0 \leqslant i \leqslant 2 m-1,(-1)^{i+1}: 0 \leqslant i \leqslant 2 m-1\right)^{t} .
$$

\section{PST on Cay $\left(D_{n}, S\right)$}

For a simple graph $\Gamma, \operatorname{Spec}(\Gamma)$ denotes the set of all eigenvalues of $\Gamma$. For any symmetric matrix $A$, assume that its eigenvalues are $\lambda_{i}(1 \leqslant i \leqslant n)$. There is a unitary matrix $P=\left(p_{1}, \cdots, p_{n}\right)$, where each $p_{i}(1 \leqslant i \leqslant n)$ is an eigenvector of $\lambda_{i}(1 \leqslant i \leqslant n)$, so that we have the following spectral decomposition of $A$

$$
A=\lambda_{1} E_{1}+\cdots+\lambda_{n} E_{n},
$$

where $E_{i}=p_{i} p_{i}^{*}(1 \leqslant i \leqslant n)$ satisfies

$$
E_{i} E_{j}=\left\{\begin{array}{cc}
E_{i} & \text { if } i=j \\
0 & \text { otherwise }
\end{array}\right.
$$

Therefore, we have the decomposition of the transfer matrix

$$
H(t)=\exp \left(-\imath \lambda_{1} t\right) E_{1}+\cdots+\exp \left(-\imath \lambda_{n} t\right) E_{n} .
$$

We also need notation of the 2-adic exponential valuation of rational numbers which is a mapping defined by

$$
v_{2}: \mathbb{Q} \rightarrow \mathbb{Z} \cup\{\infty\}, v_{2}(0)=\infty, v_{2}\left(2^{\ell} \frac{a}{b}\right)=\ell, \text { where } a, b, \ell \in \mathbb{Z} \text { and } 2 \not a b .
$$


We assume that $\infty+\infty=\infty+\ell=\infty$ and $\infty>\ell$ for any $\ell \in \mathbb{Z}$. Then $v_{2}$ has the following properties. For $\beta, \beta^{\prime} \in \mathbb{Q}$,

$(\mathrm{P} 1) v_{2}\left(\beta \beta^{\prime}\right)=v_{2}(\beta)+v_{2}\left(\beta^{\prime}\right)$;

$(\mathrm{P} 2) v_{2}\left(\beta+\beta^{\prime}\right) \geqslant \min \left(v_{2}(\beta), v_{2}\left(\beta^{\prime}\right)\right)$ and the equality holds if $v_{2}(\beta) \neq v_{2}\left(\beta^{\prime}\right)$.

Finally, we label the elements of $D_{n}=\left\langle a, b \mid a^{n}=b^{2}=1, b a b=a^{-1}\right\rangle$ as follows. For a number $u$, if $0 \leqslant u \leqslant n-1$, then $u$ corresponds to element $a^{u}$, and if $n \leqslant u \leqslant 2 n-1$, then $u$ corresponds to $b a^{u}$.

\section{1 $n$ is odd}

In this subsection, we show that if $n$ is odd and $S$ is a subset of $D_{n}$, then $\operatorname{Cay}\left(D_{n}, S\right)$ has no PST between any pair of distinct vertices.

Theorem 8. Let $n=2 m+1$ and let $S$ be a non-empty subset of $D_{n}$. Let $\Gamma=\operatorname{Cay}\left(D_{n}, S\right)$ be a connected Cayley graph with connection set $S$. Then $\Gamma$ has no PST between two distinct vertices, and $\Gamma$ is periodic if and only if it is integral and $S_{2}=\emptyset$ or $\langle a\rangle$. The minimum period of the vertices is $\frac{2 \pi}{M}$, where $M=\operatorname{gcd}\left(\lambda-\lambda_{1}: \lambda \in \operatorname{Spec}(\Gamma) \backslash\left\{\lambda_{1}\right\}\right)$.

Proof. Let $n=2 m+1$. By the above computation results, we have the following unitary matrix

$$
P=\left(p_{1}, p_{2}, p_{1}^{(1)}, p_{1}^{(2)}, p_{1}^{(3)}, p_{1}^{(4)}, \cdots, p_{m}^{(1)}, p_{m}^{(2)}, p_{m}^{(3)}, p_{m}^{(4)}\right),
$$

where

$$
p_{1}=\frac{1}{\sqrt{2 n}}(1,1, \cdots, 1)^{t}, p_{2}=\frac{1}{\sqrt{2 n}}(1, \cdots, 1,-1, \cdots,-1)^{t} .
$$

The projective matrices $E_{i}(1 \leqslant i \leqslant 2)$ are

$$
E_{1}=\frac{1}{2 n} J_{2 n}, E_{2}=\frac{1}{2 n}\left(\begin{array}{cc}
J_{n} & -J_{n} \\
-J_{n} & J_{n}
\end{array}\right)
$$

where $J_{n}$ is the all-one matrix of order $n$. For $1 \leqslant h \leqslant m$, if $\eta_{h}\left(S_{2}\right)=0$, then the associated eigenvectors are

$$
\begin{array}{ll}
p_{h}^{(1)}=\frac{1}{\sqrt{n}} \varphi_{11}^{\rho_{h}}=\frac{1}{\sqrt{n}}\left(\left\{\omega^{h k}\right\}_{k=0}^{n-1}, 0\right)^{t}, & p_{h}^{(2)}=\frac{1}{\sqrt{n}} \varphi_{21}^{\rho_{h}}=\frac{1}{\sqrt{n}}\left(0,\left\{\omega^{-h k}\right\}_{k=0}^{n-1}\right)^{t}, \\
p_{h}^{(3)}=\frac{1}{\sqrt{n}} \varphi_{12}^{\rho_{h}}=\frac{1}{\sqrt{n}}\left(0,\left\{\omega^{h k}\right\}_{k=0}^{n-1}\right)^{t}, & p_{h}^{(4)}=\frac{1}{\sqrt{n}} \varphi_{22}^{\rho_{h}}=\frac{1}{\sqrt{n}}\left(\left\{\omega^{-h k}\right\}_{k=0}^{n-1}, 0\right)^{t} .
\end{array}
$$

The corresponding projective matrices are

$$
\begin{aligned}
& E_{h}^{(1)}=\frac{1}{n}\left(\begin{array}{cc}
\Omega & 0 \\
0 & 0
\end{array}\right), \quad E_{h}^{(2)}=\frac{1}{n}\left(\begin{array}{cc}
0 & 0 \\
0 & \bar{\Omega} \\
0 & 0 \\
0 & \Omega
\end{array}\right), \quad E_{h}^{(4)}=\frac{1}{n}\left(\begin{array}{cc}
\bar{\Omega} & 0 \\
0 & 0
\end{array}\right),
\end{aligned}
$$

where $\Omega=C\left(1, \bar{\omega}^{h}, \cdots, \bar{\omega}^{(n-1) h}\right)$ is the circulant matrix with first row $\left(1, \bar{\omega}^{h}, \cdots, \bar{\omega}^{(n-1) h}\right)$. For $1 \leqslant h \leqslant m$, if $\eta_{h}\left(S_{2}\right) \neq 0$, then by Lemma 6 , the associated eigenvectors are

$$
p_{h}^{(1)}=\frac{1}{\sqrt{\iota_{h}}}\left(\overline{\ell_{h}} \varphi_{11}^{\rho_{h}}+\varphi_{21}^{\rho_{h}}\right)
$$




$$
\begin{aligned}
& =\frac{1}{\sqrt{\iota_{h}}}\left(\overline{\ell_{h}}, \overline{\ell_{h}} \omega^{h}, \cdots, \bar{\ell}_{h} \omega^{(n-1) h}, 1, \bar{\omega}^{h}, \cdots, \bar{\omega}^{(n-1) h}\right)^{t}, \\
p_{h}^{(2)} & =\frac{1}{\sqrt{\iota_{h}}}\left(\overline{\ell_{h}} \varphi_{12}^{\rho_{h}}+\varphi_{22}^{\rho_{h}}\right) \\
& =\frac{1}{\sqrt{\iota_{h}}}\left(1, \bar{\omega}^{h}, \cdots, \bar{\omega}^{(n-1) h}, \bar{\ell}_{h}, \bar{\ell}_{h} \omega^{h}, \cdots, \bar{\ell}_{h} \omega^{(n-1) h}\right)^{t}, \\
p_{h}^{(3)} & =\frac{1}{\sqrt{\iota_{h}}}\left(-\varphi_{11}^{\rho_{h}}+\ell_{h} \varphi_{21}^{\rho_{h}}\right) \\
& =\frac{1}{\sqrt{\iota_{h}}}\left(-1,-\omega^{h}, \cdots,-\omega^{(n-1) h}, \ell_{h}, \ell_{h} \bar{\omega}^{h}, \cdots, \ell_{h} \bar{\omega}^{(n-1) h}\right)^{t}, \\
p_{h}^{(4)} & =\frac{1}{\sqrt{\iota_{h}}}\left(-\varphi_{12}^{\rho_{h}}+\ell_{h} \varphi_{22}^{\rho_{h}}\right) \\
& =\frac{1}{\sqrt{\iota_{h}}}\left(\ell_{h}, \ell_{h} \bar{\omega}^{h}, \cdots, \ell_{h} \bar{\omega}^{(n-1) h},-1,,-\omega^{h}, \cdots,-\omega^{(n-1) h}\right)^{t},
\end{aligned}
$$

where $\iota_{h}=\ell_{h} \overline{\ell_{h}} n+n$ with $\ell_{h}=\sqrt{\frac{\eta_{h}\left(S_{2}^{-1}\right)}{\eta_{h}\left(S_{2}\right)}}$. The corresponding projective matrices are

$$
\begin{array}{cl}
E_{h}^{(1)}=\frac{1}{\iota_{h}}\left(\begin{array}{cc}
\bar{\ell}_{h} \ell_{h} \Omega & \bar{\ell}_{h} Q \\
\ell_{h} \bar{Q} & \bar{\Omega}
\end{array}\right), & E_{h}^{(2)}=\frac{1}{\iota_{h}}\left(\begin{array}{cc}
\bar{\Omega} & \ell_{h} \bar{Q} \\
\bar{\ell}_{h} Q & \bar{\ell}_{h} \ell_{h} \Omega
\end{array}\right), \\
E_{h}^{(3)}=\frac{1}{\iota_{h}}\left(\begin{array}{cc}
\Omega & -\bar{\ell}_{h} Q \\
-\ell_{h} \bar{Q} & \bar{\ell}_{h} \ell_{h} \bar{\Omega}
\end{array}\right), & E_{h}^{(4)}=\frac{1}{\iota_{h}}\left(\begin{array}{cc}
\bar{\ell}_{h} \ell_{h} \bar{\Omega} & -\ell_{h} \bar{Q} \\
-\bar{\ell}_{h} Q & \Omega
\end{array}\right),
\end{array}
$$

where

$$
Q=\left(\begin{array}{cccc}
1 & \omega^{h} & \cdots & \omega^{(n-1) h} \\
\omega^{h} & \omega^{2 h} & \cdots & 1 \\
\cdots & \cdots & \cdots & \cdots \\
\omega^{(n-1) h} & 1 & \cdots & \omega^{(n-2) h}
\end{array}\right)
$$

Substituting (13)-(4.8) in (9), we see that the $(u, v)$-entry of the transfer matrix is divided into two cases:

(1) $0 \leqslant u, v \leqslant n-1$ or $n \leqslant u, v \leqslant 2 n-1$. In this case,

$$
\begin{aligned}
(H(t))_{u, v}= & \frac{1}{2 n}\left(\exp \left(-\imath \lambda_{1} t\right)+\exp \left(-\imath \lambda_{2} t\right)\right) \\
& +\frac{1}{n} \sum_{h=1, \eta_{h}\left(S_{2}\right)=0}^{m}\left(\omega^{(v-u) h} \exp \left(-\imath \mu_{h} t\right)+\omega^{(u-v) h} \exp \left(-\imath \mu_{h} t\right)\right) \\
& +\sum_{h=1, \eta_{h}\left(S_{2}\right) \neq 0}^{m} \frac{1}{\iota_{h}}\left(\ell_{h} \bar{\ell}_{h} \omega^{(v-u) h}+\omega^{(u-v) h}\right) \exp \left(-\imath \mu_{h}^{(1)} t\right) \\
& +\sum_{h=1, \eta_{h}\left(S_{2}\right) \neq 0}^{m} \frac{1}{\iota_{h}}\left(\ell_{h} \bar{\ell}_{h} \omega^{(v-u) h}+\omega^{(u-v) h}\right) \exp \left(-\imath \mu_{h}^{(2)} t\right) .
\end{aligned}
$$

(2) $0 \leqslant u \leqslant n-1, n \leqslant v \leqslant 2 n-1$ or $n \leqslant u \leqslant 2 n-1,0 \leqslant v \leqslant n-1$. In this case, one can show that $|H(t)|_{u, v}<1$ and thus PST cannot occur in this case. 
We only consider the case of $0 \leqslant u, v \leqslant n-1$ as the remaining cases are analogous. By (15), we have

$$
\begin{aligned}
\left|H(t)_{u, v}\right| \leqslant & \frac{1}{2 n}\left(\left|\exp \left(-\imath \lambda_{1} t\right)\right|+\mid \exp \left(-\imath \lambda_{2} t\right)\right) \mid \\
& +\frac{1}{n} \sum_{h=1}^{m}\left(\left|\omega^{(v-u) h} \exp \left(-\imath \mu_{h}^{(1)} t\right)\right|+\left|\omega^{(u-v) h} \exp \left(-\imath \mu_{h}^{(2)} t\right)\right|\right) \\
= & \frac{2}{2 n}+\frac{1}{n} \sum_{h=1}^{m} 2 \\
= & 1 .
\end{aligned}
$$

Thus, $\left|H(t)_{u, v}\right|=1$ if and only if for $1 \leqslant h \leqslant m$ with $\eta_{h}\left(S_{2}\right) \neq 0$,

$$
\exp \left(-\imath \lambda_{1} t\right)=\frac{1}{\iota_{h}} \omega^{(u-v) h} \exp \left(-\imath \mu_{h}^{(1)} t\right)=\frac{1}{\iota_{h}} \omega^{-(u-v) h} \exp \left(-\imath \mu_{h}^{(2)} t\right)=\frac{\bar{\ell}_{h} \ell_{h}}{\iota_{h}} \exp \left(-\imath \mu_{h}^{(1)}\right) .
$$

This implies that $\iota_{h}=\bar{\ell}_{h} \ell_{h}=1$, a contradiction. Thus, we know that if there is an $h$ such that $\eta_{h}\left(S_{2}\right) \neq 0$, then there is no PST in $\Gamma$. Therefore, we consider the case of $\eta_{h}\left(S_{2}\right)=0$ for all $1 \leqslant h \leqslant\left\lfloor\frac{n-1}{2}\right\rfloor$. In this case, we know that $S_{2}=\emptyset$ or $\langle a\rangle$ by the inverse Fourier transform of the group ring $\mathbb{C}\left[\mathbb{Z}_{n}\right]$. Putting $t=2 \pi T$, we get from (15) that

$$
\begin{aligned}
& 2(u-v) \equiv 0 \quad(\bmod n), \\
& \left(\lambda_{2}-\lambda_{1}\right) T \in \mathbb{Z}, \\
& \left(\mu_{h}-\lambda_{1}\right) T \in \mathbb{Z}, 1 \leqslant h \leqslant m .
\end{aligned}
$$

Since $0=\operatorname{tr}(A)=\lambda_{1}+\lambda_{2}+4 \sum_{h=1}^{m} \mu_{h}$, from (18) and (19), we have $2 n T \in \mathbb{Z}$, and thus $T \in \mathbb{Q}$, the field of rational numbers. Now $\lambda_{1}=|S|$ is a positive integer, we know that all the eigenvalues are rational and thus integral (since they are algebraic numbers). Moreover, (17) means that $u=v$, this is to say that $\Gamma$ cannot have PST between distinct vertices when $n$ is odd.

When $u=v$, by $(15)$, we know that $\Gamma=\operatorname{Cay}\left(D_{n}, S\right)$ is period at the vertex $u$ if and only if

$$
\exp \left(-\imath \lambda_{1} t\right)=\exp \left(-\imath \lambda_{2} t\right)=\exp \left(-\imath \mu_{h}^{(1)} t\right)=\exp \left(-\imath \mu_{h}^{(2)} t\right)
$$

for all $1 \leqslant h \leqslant\left\lfloor\frac{n-1}{2}\right\rfloor$ and some $t>0$. This is equivalent to saying that $\Gamma$ is periodic at the vertex $u$ if and only if $T\left(\lambda-\lambda_{1}\right) \in \mathbb{Z}$ for all $\lambda \in \operatorname{Spec}(\Gamma)$. Equivalently, $T \operatorname{gcd}\left(\lambda-\lambda_{1}\right.$ : $\lambda \in \operatorname{Spec}(\Gamma)) \in \mathbb{Z}$. Thus, the minimum $T(>0)$ is obviously $1 / M$, where $M=\operatorname{gcd}\left(\lambda-\lambda_{1}\right.$ : $\lambda \in \operatorname{Spec}(\Gamma))$.

\section{Broglington Manifolds}

This section describes background information about Broglington Manifolds.

Lemma 9. Broglington manifolds are abundant. 
Proof. A proof is given here.

Remark 10. We note that the conclusion of Theorem 8, which says that $\Gamma$ cannot have PST when $n$ is odd, may be deduced from Godsil [24, Lemma 7.1] directly. Since every Cayley graph is vertex-transitive, if $\Gamma$ has PST, then $H(t)$ should be of the form $\zeta P$, where $\zeta$ is a unit-norm complex number and $P$ is a permutation matrix with $P^{2}=I$. Moreover, $P$ corresponds to an element of order 2 which lies in the center of $D_{n}$. When $n$ is odd, the center of $D_{n}$ is 1 . This is a contradiction. Here we just give a direct proof of the fact. Moreover using this proof, we show that if $\Gamma$ has PST, then it should be normal, namely $g^{-1} S g=S$ holds for all $g \in D_{n}$.

\section{$5.1 n$ even}

In this subsection, we assume that $n=2 m$ is even. From the discussion in the previous section, we know that in order to ensure that $\Gamma=\operatorname{Cay}\left(D_{n}, S\right)$ has PST, the set $S_{2}$ should be $\emptyset$ or $\langle a\rangle$. However, if $S_{2}=\emptyset$, then $\Gamma$ is not connected. As a consequence, $\Gamma$ has PST only if $S=\langle a\rangle$ and thus is conjugation-closed. When $\Gamma=\operatorname{Cay}\left(D_{n}, S\right)$ is normal, a necessary and sufficient condition on which $\Gamma$ has PST was provided in [13]. For completeness, we combine the results as follows.

Theorem 11. Let $n=2 m$ and let $S$ be a non-empty subset of $D_{n}$. Let $\Gamma=\operatorname{Cay}\left(D_{n}, S\right)$ be a connected Cayley graph with the connection set $S$. Then $\Gamma$ cannot have PST between two distinct vertices if $S$ is not conjugation-closed. Conversely, if $S$ is conjugation-closed, then $\Gamma$ has four eigenvalues (not necessarily dinstict) which correspond to the one-dimensional representations $\psi_{1}$ to $\psi_{4}$, respectively. One eigenvalue is $\lambda_{1}=|S|$ and the other three eigenvalues are denoted by $\lambda_{2}, \lambda_{3}, \lambda_{4}$, and some multiple eigenvalues corresponding to the two-dimensional representations $\rho_{h}$, which are denoted by $\mu_{h}(1 \leqslant h \leqslant m-1)$. Moreover, $\Gamma$ is periodic if and only if it is integral. The minimum period of the vertices is $\frac{2 \pi}{M}$, where $M=\operatorname{gcd}\left(\lambda-\lambda_{1}: \lambda \in \operatorname{Spec}(\Gamma) \backslash\left\{\lambda_{1}\right\}\right)$. Meanwhile,

(i) when $m$ is even, $\Gamma$ has PST between two distinct vertices $u$ and $v$ if and only if

(i1) all eigenvalues of $\Gamma$ are integers, namely, $\Gamma$ is integral;

(i2) $v=u+m$;

(i3) there is a constant $\alpha$ such that $v_{2}\left(\mu_{2 h^{\prime}-1}-\lambda_{1}\right)=\alpha$ for every $1 \leqslant h^{\prime} \leqslant m / 2$ and for each eigenvalue $\lambda \neq \mu_{2 h^{\prime}-1}\left(1 \leqslant h^{\prime} \leqslant m / 2\right)$, we have that $v_{2}\left(\lambda-\lambda_{1}\right)>\alpha$.

(ii) when $m$ is odd, $\Gamma$ has PST between two distinct vertices $u$ and $v$ if and only if the following conditions hold:

(ii1) all the eigenvalues of $\Gamma$ are integers;

(ii2) $v=u+m$;

(ii3) $v_{2}\left(\lambda_{3}-\lambda_{1}\right), v_{2}\left(\lambda_{4}-\lambda_{1}\right)$ and $v_{2}\left(\mu_{2 h^{\prime}-1}-\lambda\right)$ are the same for all $1 \leqslant h^{\prime} \leqslant \frac{m-1}{2}$, say, $\beta$, and $v_{2}\left(\lambda_{2}-\lambda_{1}\right), v_{2}\left(\mu_{2 h^{\prime}}-\lambda_{1}\right)$ are bigger than $\beta$ for all $1 \leqslant h^{\prime} \leqslant \frac{m-1}{2}$.

Furthermore, when the conditions hold, the minimum time at which $\Gamma$ has PST between $u$ and $v$ is $\frac{\pi}{M}$, where $M=\operatorname{gcd}\left(\lambda-\lambda_{1}: \lambda \in \operatorname{Spec}(\Gamma) \backslash\left\{\lambda_{1}\right\}\right)$.

Proof. In this situation, we have a unitary matrix

$$
P=\left(p_{1}, p_{2}, p_{3}, p_{4}, p_{1}^{(1)}, p_{1}^{(2)}, p_{1}^{(3)}, p_{1}^{(4)}, \cdots, p_{m-1}^{(1)}, p_{m-1}^{(2)}, p_{m-1}^{(3)}, p_{m-1}^{(4)}\right),
$$


where $p_{1}, p_{2}, p_{h}^{(1)}, \cdots, p_{h}^{(4)}$ and $E_{1}, E_{2}, E_{h}^{(1)}, \cdots, E_{h}^{(4)}, 1 \leqslant h \leqslant m-1$ are the same as in Subsection 4.1, and

$$
\begin{aligned}
& p_{3}=\frac{1}{\sqrt{2 n}}(1,-1,1,-1, \cdots, 1,-1)^{t}, p_{4}=\frac{1}{\sqrt{2 n}}(1,-1, \cdots, 1,-1,-1,1, \cdots,-1,1)^{t}, \\
& E_{3}=\frac{1}{2 n}\left((-1)^{u+v}\right)_{2 n \times 2 n}, E_{4}=\frac{1}{2 n}\left(e_{4}(u, v)\right)_{2 n \times 2 n},
\end{aligned}
$$

where

$$
e_{4}(u, v)= \begin{cases}(-1)^{u+v} & 0 \leqslant u, v \leqslant n-1 \text { or } n \leqslant u, v \leqslant 2 n-1 \\ (-1)^{u+v+1} & \text { otherwise. }\end{cases}
$$

As in the above section, for $1 \leqslant h \leqslant m-1$, if $\eta_{h}\left(S_{2}\right)=0$, then the associated projective matrices are the same as in (13); if there is an $h$ with $1 \leqslant h \leqslant m-1$ such that $\eta_{h}\left(S_{2}\right) \neq 0$, then by Lemma 6 , the corresponding projective matrices are the same as in (14).

We only consider the case $0 \leqslant u, v \leqslant n-1$. The $(u, v)$-entry of the transfer matrix is

$$
\begin{aligned}
(H(t))_{u, v}= & \frac{1}{2 n}\left(\exp \left(-\imath \lambda_{1} t\right)+\exp \left(-\imath \lambda_{2} t\right)+(-1)^{u+v}\left(\exp \left(-\imath \lambda_{3} t\right)+\exp \left(-\imath \lambda_{4} t\right)\right)\right) \\
& +\frac{1}{n} \sum_{h=1, \eta_{h}\left(S_{2}\right)=0}^{m-1}\left(\omega^{(v-u) h} \exp \left(-\imath \mu_{h} t\right)+\omega^{(u-v) h} \exp \left(-\imath \mu_{h} t\right)\right) \\
& +\sum_{h=1, \eta_{h}\left(S_{2}\right) \neq 0}^{m-1} \frac{1}{\iota_{h}}\left(\ell_{h} \bar{\ell}_{h} \omega^{(v-u) h}+\omega^{(u-v) h}\right) \exp \left(-\imath \mu_{h}^{(1)} t\right) \\
& +\sum_{h=1, \eta_{h}\left(S_{2}\right) \neq 0}^{m-1} \frac{1}{\iota_{h}}\left(\ell_{h} \bar{\ell}_{h} \omega^{(v-u) h}+\omega^{(u-v) h}\right) \exp \left(-\imath \mu_{h}^{(2)} t\right) .
\end{aligned}
$$

It is readily seen that if there is an $h, 1 \leqslant h \leqslant m-1$, such that $\eta_{h}\left(S_{2}\right) \neq 0$, then there is no PST in $\Gamma$. Namely, $\Gamma$ has PST only if $S_{2}=\langle a\rangle$, which means that $S$ is conjugation-closed. The rest of the theorem was proved in [13].

\section{Concluding remarks}

In this paper, we have shown that, if a Cayley graph $\Gamma=\operatorname{Cay}\left(D_{n}, S\right)$ over the dihedral group $D_{n}=\left\langle a, b \mid a^{n}=b^{2}=1, b a b=a^{-1}\right\rangle$ has PST, then $S \cap b\langle a\rangle=b\langle a\rangle$. As a consequence, $S$ is normal, namely, conjugation-closed. Since $S \cap b\langle a\rangle=b\langle a\rangle$, for $0 \leqslant i, j \leqslant n-1$, one has that $a^{i}\left(b a^{j}\right)^{-1}=a^{i-j} b=b a^{j-i} \in S$. It follows that the adjacency matrix of $\Gamma$ has the form

$$
A=\left(\begin{array}{cc}
C & J \\
J & C
\end{array}\right)
$$

where $J$ is the $n \times n$ all-one matrix and $C$ is a circulant matrix. Note that some joined graphs have the adjacency matrix as in (21), see for example, [6, Theorem 1]. However, there are some Cayley graphs over dihedral groups having PST even when the conditions of [6, Theorem 1] fail, see [13]. We leave the following questions for further research: 
Open Question 1: Determine whether there is a circulant graph Cay $\left(\mathbb{Z}_{2 n}, S\right)$ which is isomorphic to a Cayley graph over the dihedral group $D_{n}$ having PST.

Open Question 2: Determine whether circulant join graphs [6] and products or covers of graphs [19] are isomorphic to some Cayley graphs.

\section{Acknowlegemants}

The authors would like to thank the referee for his/her very careful reading and many valuable comments that helped us improve this paper. Parts of this work were done when the first author X. Cao was visiting Nanyang Technological University where he would like to express his grateful thankfulness for the hospitality. X. Cao's work is supported by the National Natural Science Foundation of China (11771007, 61572027). The work of B. Chen is supported by the National Natural Science Foundation of China Grant Numbers 11871025 and 11971175, as well as by Science and Technology Program of Guangzhou Grant Number 201804010102. The research of S. Ling was partially supported by Nanyang Technological University Research Grant M4080456.

\section{References}

[1] O.L. Acevedo, and T. Gobron, Quantum walks on Cayley graphs, J. Phys. A: Math. Gen., 39 (2006), 585-599.

[2] D. Aharonov, A. Ambainis, J. Kempe and U. Vazirani, Quantum walks on graphs, ACM Press, P. 50-59, Dec. 2000.

[3] A. Ahmadi, R. Belk, C. Tamon, C. Wendler, On mixing in continuous-time quantum walks on some circulant graphs, Quantum Comput. Inf. 3 (2003), 611-618.

[4] O. Ahmadi, N. Alon, I. F. Blake and I. E. Shparlinski, Graphs with integral spectrum, Linear Algebra Appl., 430 (2009), 547-552.

[5] B. Ahmadi, M.M.S. Haghighi and A. Mokhtar, Perfect state transfer on the Johnson scheme, arXiv:1710.09096v1, 2017.

[6] R.J. Angeles-Canul, R. Norton, M. Opperman, C. Paribello, M. Russell, C. Tamon, Perfect state transfer, integral circulants and join of graphs, Quantum Comput. Inf. 10 (2010), 325-342.

[7] L. Babai, Spectra of Cayley graphs, Jour. Combin. Theory Ser. B, 27 (1979), 180-189.

[8] M. Bašić, Characterization of circulant graphs having perfect state transfer, Quantum Inf. Process, 12(2013), 345-364.

[9] M. Bašić, M.D. Petković, Some classes of integral circulant graphs either allowing or not allowing perfect state transfer, Appl. Math. Lett., 22(2009), 1609-1615.

[10] M. Bašić, M.D. Petković and D. Stevanovic, Perfect state transfer in integral circulant graphs, Appl. Math. Lett. 22(7)(2009), 1117-1121.

[11] A. Bernasconi, C. Godsil and S. Severini, Quantum networks on cubelike graphs, Phys. Rev. Lett. 91(20)(2003), 207901. 
[12] W.G. Bridge and R. A. Mena, Rational G-matrices with rational eigenvalues, Jour. Combin. Theory Ser. A, 32 (1982), 264-280.

[13] X. Cao, K. Feng, Perfect state transfer on Cayley graphs over dihedral groups, to appear in the journal Linear and Multilinear Algebra.

[14] S. Bose, Quantum communication through a unmodulated spin chain. Phys. Rev. Lett., 91(20): 207901, 2003.

[15] A. Casaccino, S. Lloyd, S. Mancini, S. Severini, Quantum state transfer through a qubit network with energy shifts and fluctuations, Int. Jour. Quantum Inf., 7(2009), $1417-1427$.

[16] W. Cheung and C. Godsil, Perfect state transfer in cubelike graphs, Linear Algebra Appl., 435(10)(2011), 2468-2474.

[17] M. Christandl, N. Datta, T. Dorlas, A. Ekert, A. Kay and A.J. Landahl, Perfect state transfer of arbitary state in quantum spin networks, Phys. Rev. A, 73(3)(2005), 032312.

[18] M. Christandl, N. Datta, A. Ekert and A.J. Landahl, Perfect state transfer in quantum spin networks, Phys. Rev. Lett. 92(18)(2004), 187902.

[19] G. Coutinho, Quantum State Transfer in Graphs. PhD dissertation. University of Waterloo, 2014.

[20] G. Coutinho, C. Godsil, Perfect state transfer in products and covers of graphs, Linear and Multilinear Algebra, 64(2)(2016), 235-246, https://doi .org/10.1080/03081087.2015.1033381.

[21] G. Coutinho, C. Godsil, Perfect state transfer is poly-time, arXiv:1606.02264v1 [math.CO], 2016, see also: G. Coutinho, C. Godsil, Perfect state transfer is polytime, Quantum inf. Comput., 17(5 and 6), May 2017, 495-602.

[22] G. Coutinho, C. Godsil, K. Guo and F. Vanhova, Perfect state transfer on distanceregular graphs and association schemes, Linear Algebra Appl., 478(2015), 108-130.

[23] C. Godsil, Periodic graphs, Electron. J. Combin., 18(1)(2011), $\sharp 23$. arXiv:0806. 2074.

[24] C. Godsil, State transfer on graphs, Disc. Math. 312(1)(2012) 129-147.

[25] C. Godsil, When can perfect state transfer occur? Electron. J. Linear Algebra, 23(2012), 877-890.

[26] F. Harary and A. J. Schwenk, Which graphs have integral spectra? Graphs and Combinatorics (R. Bari and F. Harary, eds.), Springer-Verlag, Berlin (1974), 45-51.

[27] N. Johnston, S. Kirkland, S. Plosker, R. Storey and X. Zhang, Perfect state transfer using Hadamard diagonalizable graphs, Linear Algebra Appl., 531(2017), 373-398.

[28] W. Klotz and T. Sander, Integral Cayley graphs over abelian groups, Electronic Jour. Combin. 17(1) (2010), R81.

[29] L. Lu, Q. Huang and X. Huang, Integral Cayley graphs over dihedral groups, Jour. Algebra. Comb., https://doi .org/10.1007/s10801-017-0787-x. 
[30] P.J. McCarthy, Introduction to arithmetical functions, Universitext, Springer-Verlag, New York, 1986. https://doi.org/10.1007/978-1-4613-8620-9.

[31] M.R. Murty, Ramanujan graphs, J. Ramanujan Math. Sco. 18, No. 1(2003), 1-20.

[32] M.D. Petkovic and M. Bašić, Further results on the perfect state transfer in integral circulant graphs, Computers Math. Appl., 61(2)(2011), 300-312.

[33] J.P. Serre, Linear representations of finite groups, Springer, New York, Translated from the second French edition by L. Scott, Graduate Text in Mathematics, vol. 42, 1997.

[34] M. Štefaňák and S. Shoupý, Perfect state transfer by means of discrete-time quantum walk on complete bipartite graphs, Quantum Inf. Process, 16(3)(2017),72. https://doi .org/10.1007/s11128-017-1516-z.

[35] T.C. Silberstein, F. Scarabotti and F. Tolli, Representation Theory of the Symmetric Groups, Cambridge University Press, 2010, Cambridge studies in advanced mathematics, 121.

[36] B. Steinberg, Representation Theory of Finite Groups: An Introductory Approach. Universitext. https://doi .org/10.1007/978-1-4614-0776-8-7.

[37] Y. Tan, K. Feng, and X. Cao, Perfect state transfer on abelian Cayley graphs, Linear Algebra Appl. 563 (2019), 331-352, arXiv:1712.09260v1 [quant-ph] 26 Dec 2017.

[38] H. Zhan, An infinite family of circulant graphs with perfect state transfer in discrete quantum walks, arXiv:1707.06703v1. 